\title{
Clinical survival of indirect, anterior 3-unit surface-retained fibre-reinforced composite fixed dental prosthesis: Up to 7.5-years follow-up
}

\author{
Kumbuloglu, Ovul ; Özcan, Mutlu
}

\begin{abstract}
OBJECTIVES This prospective clinical study evaluated the performance of indirect, anterior, surfaceretained, fibre-reinforced-composite restorations (ISFRCR). METHODS Between June-2003 and January-2011, a total of 134 patients (83 females, 51 males, 16-68 years old) received 175 ISFRCRs (local ethical registration number: 14/9/4). All restorations were made indirectly on a plaster model using unidirectional E-glass fibres (everStick CB, StickTech) in combination with a laboratory resin composite (Dialogue, Schütz Dental) and cemented according to the instructions of 4 resin cements [(RelyX ARC, 3M-ESPE, n=61), Bifix DC, VOCO, n=45), Variolink II (Ivoclar Vivadent, $\mathrm{n}=32$ ) and Multilink (Ivoclar Vivadent, $\mathrm{n}=37$ )]. After baseline recordings, patients were followed at 6 months and thereafter annually up to 7.5 years. The evaluation protocol involved technical (chipping, debonding or fracture of tooth/restoration) and biological failures (caries). RESULTS Mean observation period was 58 months. Altogether, 13 failures were observed [survival rate: 97.7\%] (Kaplan-Meier). One catastrophic fracture [(cement: RelyX ARC), eight partial debonding (cement: Bifix DC (5), Multilink (1), RelyX ARC (1), Variolink II (1)] and four delaminations of veneering composite [(cement: Bifix DC (2), RelyX ARC (1), Multilink (1)] were observed. Except one replacement, all defective restorations were repaired or recemented. Annual failure rate of ISFRCRs was 1.73\%. The survival rates with the four resin cements did not show significant differences (RelyX ARC: 98.3\%; Bifix DC: 93.5\%; Variolink 2: 100\%; Multilink: 100\%) ( $\mathrm{p}=0.114)$. Secondary caries did not occur in any of the teeth. CONCLUSION The 3-unit anterior indirect surface-retained resin-bonded FRC FDPs showed similar clinical survival rate when cemented with the resin cements tested. Experienced failures in general were due to debonding of the restoration or delamination of the veneering composite. CLINICAL SIGNIFICANCE 3-unit surface retained resin-bonded FRC FDPs could be considered minimal invasive and costeffective alternatives to conventional tooth- or implant-borne FDPs. Failures were mainly repairable in the form of chipping or debonding depending on the resin cement type.
\end{abstract}

DOI: https://doi.org/10.1016/j.jdent.2015.04.006

Posted at the Zurich Open Repository and Archive, University of Zurich ZORA URL: https://doi.org/10.5167/uzh-115897

Journal Article

Accepted Version

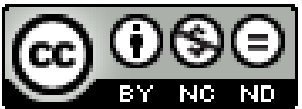

The following work is licensed under a Creative Commons: Attribution-NonCommercial-NoDerivatives 4.0 International (CC BY-NC-ND 4.0) License.

Originally published at: 
Kumbuloglu, Ovul; Özcan, Mutlu (2015). Clinical survival of indirect, anterior 3-unit surface-retained fibrereinforced composite fixed dental prosthesis: Up to 7.5-years follow-up. Journal of Dentistry, 43(6):656-663. DOI: https://doi.org/10.1016/j.jdent.2015.04.006 


\section{Clinical survival of indirect, anterior 3-unit surface-retained fiber-reinforced composite fixed dental prosthesis: Up to 7.5-years follow-up}

\section{Ovul Kumbuloglua ${ }^{\mathrm{a}}$ Mutlu Özcan ${ }^{\mathrm{b}}$}

${ }^{a}$ Ege University, School of Dentistry, Department of Prosthodontics, Izmir, Turkey.

${ }^{\mathrm{b}}$ University of Zurich, Dental Materials Unit, Center for Dental and Oral Medicine, Clinic for Fixed and Removable Prosthodontics and Dental Materials Science, Zurich, Switzerland.

Short title: Long-term clinical performance of FRC FDPs

*Part of this study has been presented at the 35th Annual Congress of European Prosthodontic Association (EPA), September, 29th, 2011, Bern, Switzerland.

*Corresponding author. Prof. Dr.med.dent. Mutlu Özcan, Ph.D, Head of Dental Materials Unit, University of Zürich, Center for Dental and Oral Medicine, Clinic for Fixed and Removable Prosthodontics and Dental Materials Science, Plattenstrasse 11, CH-8032, Zürich, Switzerland. Tel: +41 4463 45600; Fax: +41 4463 44305; E-mail: mutluozcan@hotmail.com 


\section{ABSTRACT}

Objectives: This prospective clinical study evaluated the performance of indirect, anterior, surface-retained, fiber-reinforced-composite restorations (ISFRCR).

Methods: Between June-2003 and January-2011, a total of 134 patients (83 females, 51 males, 16-68 years old) received 175 ISFRCRs (Local ethical registration number: 14/9/4). All restorations were made indirectly on a plaster model using unidirectional E-glass fibers (everStick C\&B, StickTech) in combination with a laboratory resin composite (Dialog, Schütz Dental) and cemented according to the instructions of 4 resin cements [(RelyX ARC, 3M-ESPE, n=61), Bifix DC, VOCO, n=45), Variolink II (Ivoclar Vivadent, $n=32$ ) and Multilink (Ivoclar Vivadent, $\mathrm{n}=37$ )]. After baseline recordings, patients were followed at 6 months and thereafter annually up to 7.5 years. The evaluation protocol involved technical (chipping, debonding or fracture of tooth/restoration) and biological failures (caries).

Results: Mean observation period was 58 months. Altogether, 13 failures were observed [survival rate: 97.7\%] (Kaplan-Meier). One catastrophic fracture [(cement: RelyX ARC), eight partial debonding (cement: Bifix DC (5), Multilink (1), RelyX ARC (1), Variolink II (1)] and four delaminations of veneering composite [(cement: Bifix DC (2), RelyX ARC (1), Multilink (1)] were observed. Except one replacement, all defective restorations were repaired or recemented. Annual failure rate of ISFRCRs was $1.73 \%$. The survival rates with the four resin cements did not show significant differences (RelyX ARC: 98.3\%; Bifix DC: 93.5\%; Variolink 2: 100\%; Multilink: 100\%) ( $p=0.114)$. Secondary caries did not occur in any of the teeth.

Conclusion: The 3-unit anterior indirect surface-retained resin-bonded FRC FDPs showed similar clinical survival rate when cemented with the resin cements tested. Experienced failures in general were due to debonding of the restoration or delamination of the veneering composite.

Clinical Trial Registration Number: NCT02343796.

Keywords: Clinical study, Fiber Reinforced Composites, Resin cements, Survival 


\section{Introduction}

Adhesion of resin-based materials to enamel and dentin, and developments in resin composites transformed the application of invasive therapy options to minimal invasive ones in reconstructive dentistry. Missing anterior teeth can be restored with a wide range of therapy options such as partial removable dentures, resin-bonded fixed dental prosthesis (FDP) made of metal-ceramic, all ceramic, fiber reinforced composite (FRC), conventional full coverage FDPs, or implants. Each of these techniques has both advantages and disadvantages, and some of the latter may include overall complexity, with biological and financial costs, and cause difficulties in maintaining oral hygiene. Full coverage FDPs present the longest track but this indication requires abutment tooth preparation yielding to irreversible biological costs. ${ }^{1}$ On the other hand, indication of implant-supported FDPs certainly increased dramatically over the last two decades but the incidence of biological complications such as peri-implantitis and its therapy has not been resolved yet. ${ }^{2}$ Among the minimal invasive options, studies on metal-ceramic resin-bonded FDPs reported debonding at the metal-composite cement or enamel-composite cement interface. ${ }^{3,4}$ Fractures and debondings were more commonly reported with all ceramic resin-bonded FDPs as the main technical complications. ${ }^{5}$ In addition, favourable occlusion accompanied with sufficient space for the metal are required in order to minimize the direct stress on the FDP. ${ }^{3,4}$

Recently, the use of FRC applications has greatly increased in dentistry in the form of root posts, ${ }^{6}$ periodontal splints, ${ }^{7}$ or bonded FDPs. ${ }^{8-12}$ Structurally, an FRC is composed of two components namely, the resin matrix that serves as carrier and protector of the fibers and the specifically oriented fibers with the purpose of improving the mechanical properties of resin composite. Ultrahigh molecular weight polyethylene, E-glass or S-glass, aramid (Kevlar) and carbon fibers are the most frequently used FRC materials for the construction of FDPs. ${ }^{13}$ Among different fiber materials, silanized and preimpregnated glass fibers are more commonly used because of their favourable optical properties, possibility to withstand tensile stresses and 
prevent crack propagation in resin matrix. ${ }^{14,15}$ When fabricating FRC FDPs, reinforcement with long unidirectional fibers at the tensile side of the construction is recommended. ${ }^{14,15}$ Such FDPs can be fabricated either directly in the mouth or indirectly by a dental technician. When compared with the direct technique, the indirect technique offers ease of working, a higher degree of conversion rate for the veneering composite and a better surface finish. ${ }^{16}$ In the case of surface-retained applications, indirect FRC FDPs are then bonded directly onto enamel and in the case of retentive ones, they are bonded to the cavities that have margins in enamel/dentin complex.

Studies on clinical performance of resin-bonded FRC FDPs are limited. Overall survival rate of $75 \%$ and functional survival rate of $93 \%$ were reported after a follow-up period of 24 to 63 months. ${ }^{8-12}$ One systematic review on clinical survival of commercially available different FRC products without discrimination between type of retainers, fabrication technique, type of fibers, cements, preparation designs, have been published. ${ }^{17}$ This systematic review summarized the survival rate of FRC FDPs based on a limited number of clinical studies, all of which reported relatively short follow-up results. Problems associated with commonly used FRC systems included fractures, wear and discoloration of the veneering resin composite. ${ }^{17}$ Besides clinical studies, numerous in vitro studies have been carried out to measure the fracture strength of FRC FDPs under mechanical loads. ${ }^{15,18-23}$ In these investigations, most of the failure types were noted at the interface between the fiber and the resin matrix at the loading point (pontic), and at the connectors. Cohesive delamination within the veneering resin was also found to be the most common failure mode both in in vitro and clinical studies. To the authors ' best knowledge, in none of these studies cement type was of focus.

The objectives of this study therefore were to evaluate the clinical performance of indirect, anterior, surface-retained, FRC FDPs bonded using four different resin cements. The null hypothesis tested was that the cement type would not affect the survival of FRC FDPs. 


\section{Materials and methods}

\subsection{Study design}

The brands, types, manufacturers and chemical compositions of the materials used in this study are listed in Table 1.

\subsection{Inclusion and exclusion criteria}

As of January 2003, FRC FDPs are offered as alternative to other conventional therapy options at the Department where the study was conducted. Between June-2003 and January-2011, 134 patients aged between 16 and 68 years old ( 83 females, 51 males, mean age: 42) referred to the Department of Prosthodontics, Dental School Ege University, Izmir (Turkey) from other Departments, received 175 indirect anterior FRC FDPs. Before enrolment in the trial, all patients were provided with a written informed consent form approved by the ethical committee of the university institutional review board (Vote number of the local Ethical Committee no: 7OM/743; Local ethical registration number: 14/9/4; Clinical Trial Registration Number: NCT02343796). Information was given to each patient regarding the alternative treatment options. The inclusion criteria were as follows: all subjects were required to be at least 18 years old, able to read and sign the informed consent document, physically and psychologically able to tolerate conventional restorative procedures, having no active periodontal or pulpal diseases, having no primary caries, not allergic to resinbased materials, not pregnant or nursing, having antagonist teeth opposing the FRC FDP to be restored, willing to return for follow-up examinations as outlined by the investigators. The patients who had more absent teeth than the tooth to be replaced in the rest of the dentition or those having diastemas were not excluded.

2.3 Clinical procedures, restoration design and fabrication

All FDPs replaced one missing tooth and bonded to two adjacent abutment teeth were used for retention. No cantilever FDPs were involved. 
Treatment was performed in two sessions: 1) Impression and model analysis, 2) Try-in, cementation and polishing of the FDP. Initially, impressions were made using polyvinyl siloxane material (Affinis, Coltène/Whaledent AG, Altstätten, Switzerland), poured in plaster for model analysis.

FRC FDPs were made in one dental laboratory on the full arch plaster cast. The model was first isolated with two coats of isolation medium. One unidirectional pre-impregnated E-glass fiber bundle (everStick C\&B, StickTech Ltd., Turku, Finland) was used to reinforce the veneering composite. Each bundle consists of about 4000 glass fibers, with a diameter of $1.7 \mathrm{~mm}$, embedded in a PMMA/bis-GMA matrix. Before the fibers were placed on the cast, a thin layer of flowable composite (Grandio Flow, Voco, Cuxhaven, Germany) was applied on the lingual surface of the abutment teeth. The fiber bundle was placed in the bed of the flowable resin using metal hand instruments. Dimension of the retainer wings varied depending on the mesio-distal and cervico-incisal length as well as the position of the cingulum palatally but principally the width of the FDP was dictated by the width of the fiber bundle, not exceeding 2 to $3 \mathrm{~mm}$. The length of the retainer varied between 6 to $8 \mathrm{~mm}$, again depending on the available area palatally, FRC starting from mesial side of each abutment teeth but not exceeding the distal marginal ridge, positioned above the cingulum, towards the incisal edge.

After photo-polymerization with a halogen polymerization unit (light output: $1200 \mathrm{mw} / \mathrm{cm}^{2}$ ), the framework was veneered with a resin composite (Dialog, Schütz Dental, Rosbach, Germany). An opaque resin was used in some cases to improve the optical properties. The veneering resin composite was built incrementally; the construction was polymerized for the final time with a heat-light polymerization oven (Visio Beta, 3M ESPE, Seefeld, Germany).

In the second treatment session, the abutment teeth were cleaned with pumice. The fit of the FDP was checked using a silicon material (Fit Checker, GC, Tokyo, Japan), bonding surfaces were cleaned with pumice after try-in; if needed adjustments were made at low speed using diamond burs. Rubber-dam was 
applied where possible. Thirty per cent $(n=53)$ of the FDPs were cemented under rubber dam. Meanwhile, the cementation surface of the FDP was treated with resin monomer of the corresponding resin cement using a microbrush (Adper Scotchbond for RelyX ARC, Futurabond DC for Bifix DC, Heliobond for Variolink II and Multilink Primer A and B for Multilink). The resin was left unpolymerized, shielded from light, for at least 5 minutes to allow the resin to penetrate and activate the IPN-phase of the polymethacrylate polymer matrix of the FRC framework.

The bonding surface of the abutment teeth was acid-etched with $37 \%$ phosphoric acid gel for $60 \mathrm{~s}$, rinsed thoroughly for at least $30 \mathrm{~s}$ and gently air dried for $5 \mathrm{~s}$. FDPs were luted with one of the following resin cement: RelyX ARC (3M ESPE, Seefeld, Germany), Bifix DC (VOCO, Cuxhaven, Germany), Variolink II (Ivoclar Vivadent, Schaan, Liechteinstein) and Multilink (Ivoclar Vivadent) according to each manufacturer's instructions. After removal of excess material, the resin composite cement was photo-polymerized for $20 \mathrm{~s}$ from 4 surfaces (lingual, labial, mesial, distal) (Astralis 10, SN 018766, Ivoclar Vivadent, light output: 1200 $\mathrm{mW} / \mathrm{cm}^{2}$ ). The power output of the unit was measured with a radiometer (Cure-Rite, Dentsply-Caulk, Milford, USA) before the placement of each restoration. After polymerization, restoration margins were finished. Occlusion was adjusted with fine diamond burs (60- and 40- $\mu \mathrm{m}$ grit) and finally, the restoration was polished with pointed silicon polishers (Astropol, Ivoclar Vivadent) and abrasive polishing brushes (Astrobrush). Patients received individual instructions to maintain plaque control.

One operator applied all the restorations who has experience in adhesive dentistry ( $>15$ years since graduation). No randomization was done and the resin cements were used depending on their availability in our clinics. 


\subsection{Evaluation}

The evaluation protocol involved technical (chipping, debonding or fracture of tooth/restoration) and biological failures (caries). After baseline recordings, patients were followed at 6 months and thereafter annually up to 7.5 years. Patients were instructed to call upon experience of a failure.

Chipping failures were repaired using the CoJet System (3M ESPE). The surfaces with delamination were cleaned with pumice, air-abraded with alumina particles coated with silica (CoJet Sand, 3M ESPE). Silane coupling agent (ESPE-Sil, 3M ESPE) was applied, waited for 1 minute for its reaction. An adhesive resin (Heliobond, Ivoclar Vivadent, Schaan, Liechtenstein) was applied with microbrush, air-thinned and photo polymerized for $20 \mathrm{~s}$. Then a repair composite (Tetric ceram, Ivoclar Vivadent, Schaan, Liechtenstein) was applied and photo polymerized for $40 \mathrm{~s}$ from each direction. The debonded FDPs were recemented using the same cement with the original one.

2.5 Statistical analysis

Survival analyses were performed with statistical software program (SPSS 14.0; SPSS Inc, Chicago, IL, USA) using Kaplan-Meier and Log Rank (Mantel-Cox) tests to obtain the cumulative survival rates in relation to observation time. Cox regression analysis was used to estimate the experienced failures attributable to location, missing tooth type, and application of rubberdam. $P$ values less than 0.05 were considered to be statistically significant in all tests. Based on the survival rates, Log Rank sample size analysis was performed (MedCalc 13 software, Ostend, Belgium) to compare the survival rates, calculate the power of this study and suggest sample size for future studies.

\section{Results}

Mean observation period was 58 months. Sixty-three FRC FDPs were placed in the maxilla, and 112 on the mandible. FDPs replaced 78 central and 97 lateral incisors as pontics [(RelyX ARC, 3M-ESPE, n=61), Bifix 
DC, VOCO, n=45), Variolink II (Ivoclar Vivadent, $n=32$ ) and Multilink (Ivoclar Vivadent, $n=37$ )]. Distribution of FRC FDPs is presented in Table 2. In total, 9 recalls ( 6 months and annual after baseline) were performed after baseline measurements. No drop out was experienced with a recall rate of $100 \%$ after the mean observation period of 58 months.

Secondary caries, endodontic complications, tooth fracture were not observed in any of the restored teeth.

Altogether, 13 failures were observed at a mean follow-up of 58 months [survival rate: $97.7 \%$ ] (KaplanMeier) (Fig. 2). One catastrophic fracture [(cement: RelyX ARC), eight partial debonding (cement: Bifix DC (5), Multilink (1), RelyX ARC (1), Variolink II (1)] and four delaminations (chipping) of veneering composite [(cement: Bifix DC (2), RelyX ARC (1), Multilink (1)] were observed. The fracture occurred in the connector area without cohesive fracture of the FRC.

Except one replacement, all defective restorations were repaired or recemented and remained functional until the final observation date. In the replacement case, other treatment options were proposed and upon patient's request, again same type of FRC FDP was constructed and cemented. Partial debondings $(n=8)$ showed exclusively adhesive failures between the cement and enamel.

Annual failure rate of FRC FDPs was $1.73 \%$. The survival rates with the four resin cements did not show significant differences (RelyX ARC: 98.3\%; Bifix DC: 93.5\%; Variolink II: 100\%; Multilink: 100\%) ( $p>0.05)$ (Kaplan-Meier, Log Rank; Mantel-Cox).

No significant difference was found between maxilla and mandible ( $p>0.05)$ and central or lateral incisors $(p>0.05)$ or the cementation under rubberdam or not $(p>0.05)$ (Cox regression analysis).

When two extreme groups were compared (Bifix DC vs. Variolink II) with the survival rate approximated from 1 to 0.999 , power of this study was calculated as $53.6 \%$. Again, when two extreme groups were compared (Bifix DC vs. Variolink II) and assuming that future studies would have equal number of subjects 
in both groups, with the survival rate approximated from 1 to 0.999 , sample size needed to detect a significant difference $(p<0.05)$ between the two groups with a power of $80 \%$ was calculated as 127 in each group.

\section{Discussion}

This study evaluated the clinical performance of indirect, anterior, surface-retained, FRC FDPs cemented using four different resin cements. Based on the results of this study, no significant difference was found between survival rates of restorations bonded with different resin cements, yielding to acceptance of the null hypotheses.

The majority of the similar studies on FRC FDPs presented a survival rate of $\geq 72 \%$ after 2 to 5 years. ${ }^{17}$ Such results could be considered rather modest to define FRC FDPs as permanent restorations when only success rates are considered. However, in the majority of the studies, repair options were considered to increase the survival rate of the restorations. Based on this, FRC FDPs are classified as semi-permanent restoration options in reconstructive dentistry. In fact, as a whole, there are several advantages of direct or indirect FRC FDP techniques as opposed to other reconstruction options. Firstly, the biological cost is low since little or no tooth tissues need to be removed, and consequently all future treatment options remain available. The procedure can be completed in single or two visits, and often no temporization is required. This also gives the advantage of profiting maximum from adhesive techniques, as the enamel/dentin are not contaminated with any kind of temporary material. Also, the clinician has complete control over the shade and shape of the pontic, and because the prosthesis metal-free, there is no aesthetic problem with metal showing through thin abutment teeth. Furthermore, material costs are low, and there is no laboratory fee when the clinician makes restoration. Repairs, additions and colour changes are also easily performed by the clinician chairside. ${ }^{23}$ 
One comparable treatment option is metal-ceramic resin-bonded FDPs. An analysis of 60 studies on resin-bonded FDPs revealed a survival rate of $74 \%$ after 4 years. ${ }^{3}$ Repeated stresses can predispose fatigue failures of the adhesive joint between the metal framework and the tooth surface. By selecting materials with a lower modulus of elasticity than those of cast metal alloys such as FRCs or resin composites, similar flexural properties of the bonded joint and thereby less deflexion of the restorations can be expected. ${ }^{24}$ Differences between survival rates in different studies on metal-ceramic FRC FDPs are usually attributed to variation in patient selection, tooth preparation, choice of materials, luting cements, and operator's experience. . $^{3,4}$

An analysis with mean observation period of 58 months could be considered medium term follow-up. Yet, failure types even at this stage could indicate some possible complications in the future. The most common failure type experienced was debonding followed by cohesive delamination in the veneering composite, and complete fracture of the FRC FDP. The low incidence of complete fracture could be attributed to the FRC type used. Factors affecting the durability of FRC restorations include the properties of the fibers, polymer matrix, impregnation of fibers with the resin, adhesion of fibers to the matrix, the quantity of fibers, and the direction, orientation, location, construction, distribution, and position of the fibers. ${ }^{25}$ In this study, due to favourable in vitro observations, a single longitudinal E-glass fiber bundle was used in the framework and no supporting fibers for the pontic were added. ${ }^{18-22,24,25}$ Higher quantity of fibers in the pontic prevents veneer delaminations. Similar observations were made with different fibers and veneering composites in previous studies. ${ }^{18-22}$ Recent data on finite element modelling of FRC FPDs suggests ways to optimize the design and provide better support for the pontic with lower interfacial stress between veneering composite and fiber framework. ${ }^{20,24}$ Moreover, in FDP constructions the connector areas have to resist the highest tensile and shear forces. ${ }^{24}$ 
One reason for the chipping in the veneering composite could be attributed to the presence of unsupported areas under the veneering composite yielding to its cohesive fracture. One other reason could be simply due to the cohesive strength of the veneering composite itself. Nevertheless, such failures are easy to repair chairside. However, future studies should observe the chipping phenomena of such restorations by adding more fibers in the pontic area.

The most common failure type observed in this study was debonding regardless of the cement type used. Since the adhesive surface of the FRCs was resin composite, one would expect debonding or dislodgement from the resin composite luting agent to be a minor problem. In spite of that, debondings were observed in this study. It is known that the debonding at the interface between the abutment teeth and the retainer represents a major mode of failure, though the initiation, progression of a crack between the cement and tooth or cement and restorative material substrates, and the pattern of debonding remain unclear. ${ }^{3,4}$ No randomization could be employed as regards to the choice of cement as the purchase decision for the cements were made in the department based on the economical aspects. Although resin cements showed chemical variations no significant difference observed in this study could be explained on the grounds that enamel was etched in all cases. Similarly, the predominant reason for failure of surface retained metalceramic resin-bonded FDPs is known to be debonding. ${ }^{3,4}$ The design of the clinical protocol in this study, did not involve preparation of any cavities or undercuts. In this kind of an approach, retention of the FDP was mainly achieved using the undercut palatal concavity of the abutment teeth. In principle surface-retained FDPs represent the worse case scenario due to the lack of mechanical retentive elements. In most of the cases, the cement remained on the cementation surface of the FDPs indicating an adhesive failure between the enamel and the cement. Even though excellent adhesion is achieved on enamel, apparently torque forces exceeded the adhesive forces between the cement and the enamel. Interestingly however, after secondary cementation no failures were observed up until final observation period. It also has to be noted 
that in this study, only thirty per cent of the FRC FDPs were placed under rubberdam, which did not affect the outcome significantly. Whether FDPs cemented without using rubberdam survive less, requires more attention in reporting long-term results. Primary outcome measure of this study was the functional durability with such restorations. Economical reasons favoured this material choice for the FDPs against other therapy options. Thus, qualitative analysis related to wear and discoloration parameters using USPHS criteria were not considered. Methacrylate based resin composites versus ceramic materials in randomized clinical trials show more surface quality changes due to wear and discoloration that require more maintenance. ${ }^{29,30}$ Such surface degradation was reported to be higher particularly in the presence of biofilm ${ }^{31}$ which needs to be communicated with the patient when composite based restorations are indicated.

Recently, more favourable results are reported with cantilever design. ${ }^{5,26,27}$ In this study, in order to benefit from adhesion to enamel in a larger surface area, two retainers were used. Annual failure rate of $1.73 \%$ with functional survival rates ranging between 93.5 and $100 \%$ in this study, were slightly higher than those reported in the systematic review on FRC FDPs. ${ }^{17}$ This systematic review reported substantial heterogeneity between studies to make estimates for survival rates at 2 year of follow-up as it was not possible to build a reliable regression model to indicate risk factors. Yet, the Kaplan-Meier estimate of the overall survival, based on the data from 435 patients in 15 studies was $73.4 \%$ at 4.5 years. Observation duration of 58 months in this study could be considered short in this regard. The technical problems such as fracture of the FRC FDP and delamination of the veneering composite were the most commonly observed ones. When single studies were evaluated, similar failure types were observed in a multicenter study at 5 years with success rate of $71.2 \%$ and survival rate of $77.5 \%$ where the restoration types showed heterogeneity of inlay, hybrid and surface-retained posterior FPDs, where the latter showed more debonding. ${ }^{31}$ As for anterior surface-retained FRC FDPs, again in a multicenter study where more operators were involved, at 5 years, survival rate of $64 \%$ and success rate of $45 \%$ was reported with median survival time 58 months. ${ }^{11}$ For 
surface-retained FDPs, additional mechanical retention did not improve survival significantly. Due to the heterogeneity of the study on posterior FRC FDPs, ${ }^{31}$ at this moment it cannot be stated that anterior ones show less survival rate. Limited information is available on the long-term survival of all-ceramic resin-bonded FDPs but ten-year survival of such anterior FDPs made of In-Ceram with two retainers $(n=38$; mean observation: 120 months) showed $73.9 \%$, whereas those with one retainer $(n=22$; mean observation: 111 months) $94.4 \%$ survival. ${ }^{5}$ Such long-term evaluation with FRC FDPs is currently not available but one retainer design warrants further clinical investigation.

The rapid development and relatively short clinical history of the current resin-bonded FRC FDP technique have given rise to concerns on the through evaluation of this procedure. However, situations where implant prognosis is questionable, young patients due to biological price of the dental tissues or patients with economical limitations could be offered such an alternative which was also reported to increase oral health related quality of life. ${ }^{32}$

\section{Conclusions}

1. The 3-unit anterior indirect surface-retained resin-bonded FRC FDPs showed similar clinical survival rate when cemented with the resin cements tested.

2. Experienced failures in general were due to debonding of the restoration or delamination of the veneering composite.

\section{Conflict of interest}

The authors did not have any commercial interest in any of the materials used in this study. 


\section{REFERENCES}

1. Pjetursson BE, Tan K, Lang NP, Brägger U, Egger M, Zwahlen M. A systematic review of the survival and complication rates of fixed partial dentures (FPDs) after an observation period of at least 5 years. Clinical Oral Implants Research 2004;15:625-642.

2. Faggion CM Jr, Listl S, Tu YK. Assessment of endpoints in studies on peri-implantitis treatment-a systematic review. Journal of Dentistry 2010;38:443-450.

3. Creugers NHJ, Snoek PA, Hof van't MA. Clinical performance of resin-bonded bridges: a 5-year prospective study. I. Design of the study and influence of experimental variables. Journal Oral Rehabilitation 1989;16:427-436.

4. Creugers NHJ, Hof van't MA. An analysis of clinical studies on resin-bonded bridges. Journal of Dental Research 1991;70:146-149.

5. Kern M, Sasse M. Ten-year survival of anterior all-ceramic resin-bonded fixed dental prostheses. Journal of Adhesive Dentistry 2011;13:407-410.

6. Lassila LV, Tanner J, Le-Bell AM, Narva K, Vallittu PK. Flexural properties of fiber reinforced root canal posts. Dental Materials 2002;20:29-36.

7. Kumbuloglu O, Saracoglu A, Özcan M. Pilot study of unidirectional E-glass fibre-reinforced composite resin splints: up to 4.5-year clinical follow-up. Journal of Dentistry 2011;39:871-877.

8. Monoca C, Ferrari M, Miceli GP, Scotti R. Clinical evaluation of fiber-reinforced composite inlay FPDs. International Journal Prosthodontics 2003;16:391-325.

9. Vallittu PK. Survival rates of resin-bonded, glass fiber-reinforced composite fixed partial dentures with a mean follow-up of 42 months: a pilot study. Journal of Prosthetic Dentistry 2004;91:241-246. 
10. Göhring TN, Roos M. Inlay-fixed partial dentures adhesively retained and reinforced by glass fibers: clinical and scanning electron microscopy analysis after five years. European Journal of Oral Sciences 2005;113:60-69.

11. Céleste CM, van Heumen CM, van Dijken J, Tanner J, Pikaard R, Lassila L, Creugers N, Vallittu K, Kreulen CM. Five-year survival of 3-unit fiber-reinforced composite fixed partial dentures in the anterior area. Dental Materials 2009;25:820-827.

12. Wolff D, Schach C, Kraus T, Ding P, Pritsch M, Mente J, Joerss D, Staehle HJ. Fiber-reinforced composite fixed dental prostheses: a retrospective clinical examination. Journal Adhesive Dentistry 2011;13:187-194.

13. Vallittu PK. Flexural properties of acrylic resin polymers reinforced with unidirectional and woven glass fibers. Journal of Prosthetic Dentistry 1999;81:318-326.

14. Dyer S, Lassila L, Vallittu P. Effect of cross-sectional design on the modulus of elasticity and toughness of fiber-reinforced composite materials. Journal of Prosthetic Dentistry 2005;94:219-226.

15. Fennis WM, Tezvergil A, Kuijs RH, Lassila LV, Kreulen CM, Creugers $\mathrm{NH}$, et al. In vitro fracture resistance of fiber reinforced cusp-replacing composite restorations. Dental Materials 2005;21:565-572.

16. Souza RO, Özcan M, Michida SM, de Melo RM, Pavanelli CA, Bottino MA, Soares LE, Martin AA. Conversion degree of indirect resin composites and effect of thermocycling on their physical properties. Journal of Prosthodontics 2010;19:218-225.

17. van Heumen CCM, Kreulen CM, Creugers NHJ. Clinical studies of fiber-reinforced resin-bonded fixed partial dentures: a systematic review. European Journal of Oral Sciences 2009;117:1-6.

18. Kumbuloglu O, Özcan M, User A. Fracture strength of direct surface-retained fixed partial dentures: effect of fiber reinforcement versus the use of particulate filler composites only. Dental Materials Journal 2008;27:195-202. 
19. Özcan M, Breuklander MH, Vallittu PK. The effect of box preparation on the strength of glass fiberreinforced composite inlay-retained fixed partial dentures. Journal of Prosthetic Dentistry 2005;93:337-345.

20. Özcan M, Koekoek W, Pekkan G. Load-bearing capacity of indirect inlay-retained fixed dental prostheses made of particulate filler composite alone or reinforced with E-glass fibers impregnated with various monomers. Journal of the Mechanical Behavior of Biomedical Materials 2012;12:160-167.

21. Li W, Swain MV, Li Q, Ironside J, Steven GP. Fibre reinforced composite dental bridge. Part I. Experimental investigation. Biomaterials 2004;25:4987-4993.

22. Xie Q, Lassila LV, Vallittu PK. Comparison of load-bearing capacity of direct resin-bonded fiberreinforced composite FPDs with four framework designs. Journal of Dentistry 2007;35:578-582.

23. Aktas G, Basara EG, Sahin E, Uctasli S, Vallittu PK, Lassila LV. Effects of different cavity designs on fracture load of fiber-reinforced adhesive fixed dental prostheses in the anterior region. Journal of Adhesive Dentistry 2013;15:131-135.

24. Brendeke J, Özcan M. Effect of physicochemical aging conditions on the composite-composite repair bond strength. Journal of Adhesive Dentistry 2007;9:399-406.

25. Yokoyama D, Shinya A, Lassila LVJ, Gomi H, Nakasone Y, Vallittu PK, et al. Main framework design of anterior fiber reinforced hybrid composite fixed partial denture: 3D finite element study. International Journal Prosthodontics 2009;22:405-412.

26. Vallittu PK. Strength and interfacial adhesion of FRC-tooth system. In: P.K. Vallittu, Editor, The Second International Symposium on Fibre-Reinforced Plastics in Dentistry Symposium Book on the Scientific Workshop on Dental Fibre-Reinforced Composite on 13 October 2001 Nijmegen, The Netherlands (2002).

27. Anderson JD. Ten-year survival rate for cantilevered fixed partial dentures. Evidence Based Dentistry 2005;6:96-97. 
28. van Dalen A, Feilzer AJ, Kleverlaan CJ. In vitro evaluation of failure loads of nonmetal cantilevered resin-bonded fixed dental prostheses. Journal of Adhesive Dentistry 2008;10:461-469.

29. Meijering AC, Creugers NH, Roeters FJ, Mulder J. Survival of three types of veneer restorations in a clinical trial: a 2.5-year interim evaluation. Journal of Dentistry 1998;26:563-568.

29. Gresnigt MM, Kalk W, Özcan M. Randomized clinical trial of indirect resin composite and ceramic veneers: up to 3-year follow-up. Journal of Adhesive Dentistry 2013;15:181-190.

30. Rinastiti M, Özcan M, Siswomihardjo W, Busscher HJ, van der Mei HC. Effect of biofilm on the repair bond strengths of composites. Journal of Dental Research 2010;89:1476-1481.

31. van Heumen CC, Tanner J, van Dijken JW, Pikaar R, Lassila LV, Creugers NH, Vallittu PK, Kreulen CM. Five-year survival of 3-unit fiber-reinforced composite fixed partial dentures in the posterior area. Dental Materials 2010;26:954-960.

32. Anweigi L, Finbarr Allen P, Ziada H. Impact of resin bonded bridgework on quality of life of patients with hypodontia. Journal of Dentistry 2013;41:683-688. 


\section{Captions to the tables and legends:}

\section{Tables:}

Table 1. The brand, type, manufacturer, and chemical composition of the main materials used in this study. Table. 2. Distribution and location of the FRC FDPs cemented using 4 types of resin cement.

\section{Legends:}

Figs. 1a-c Representative photos of a) Missing 41, b) indirect FRC FDP replacing the missing tooth at baseline, c) FRC FDP at 3 years follow up.

Fig. 2 Event-free survival rates of surface-retained, anterior FRC FDPs based on the resin cement type. (RelyX ARC, n=61, Bifix DC, n=45, Variolink II, n=32, Multilink, n=37). 
Tables:

\begin{tabular}{|c|c|c|c|}
\hline Brand & Type & Manufacturer & Chemical composition \\
\hline RelyX ARC & $\begin{array}{l}\text { Dual- } \\
\text { polymerized } \\
\text { cement }\end{array}$ & $\begin{array}{l}\text { 3M ESPE, Seefeld, } \\
\text { Germany }\end{array}$ & $\begin{array}{l}\text { bis-GMA, TEGDMA polymer } \\
\text { zirconia/silica filler ( } 68 \mathrm{wt} \%) \\
\text { average filler particle size: approximate } \\
1.5 \mathrm{~mm} \\
\text { Paste A: approx. } 68 \mathrm{wt} \% \text { zirconia/silica } \\
\text { fillers } \\
\text { Paste B: approx. } 67 \mathrm{wt} \% \\
\text { zirconia/silica fillers }\end{array}$ \\
\hline Bifix DC & $\begin{array}{l}\text { Dual- } \\
\text { polymerized } \\
\text { cement }\end{array}$ & Voco, Cuxhaven, Germany & $\begin{array}{l}\text { bis-GMA (20-22 wt\%) dimethacrylates, } \\
\text { silicate fillers }\end{array}$ \\
\hline
\end{tabular}

\begin{tabular}{|lll}
\hline Variolink II & $\begin{array}{l}\text { Dual- } \\
\text { polymerized } \\
\text { cement }\end{array}$ & $\begin{array}{l}\text { Ivoclar Vivadent AG, } \\
\text { Schaan, Liechtenstein }\end{array}$
\end{tabular}

monomer matrix: bis-GMA, urethane

dimethacrylate, triethylene glycol

dimethacrylate

inorganic fillers: barium glass, ytterbium

trifluoride, Ba-Al-fluorosilicate glass,

spheroid mixed oxide

Mean particle size: $0.7 \mu \mathrm{m}$

\begin{tabular}{|c|c|c|c|}
\hline Multilink & $\begin{array}{l}\text { Self-adhesive } \\
\text { cement }\end{array}$ & Ivoclar Vivadent AG & $\begin{array}{l}\text { Monomer matrix: dimethacrylate, HEMA } \\
\text { Inorganic fillers: barium glass, ytterbium } \\
\text { trifluoride, spheroid mixed oxide. } \\
\text { Mean particle size: } 0.9 \mu \mathrm{m} \\
\text { Inorganic fillers: approx. } 40 \mathrm{v} \%\end{array}$ \\
\hline
\end{tabular}

everStick C\&B E-glass fiber StickTech Ltd., Turku, Finland

E-glass fibre/PMMA/

bis-GMA

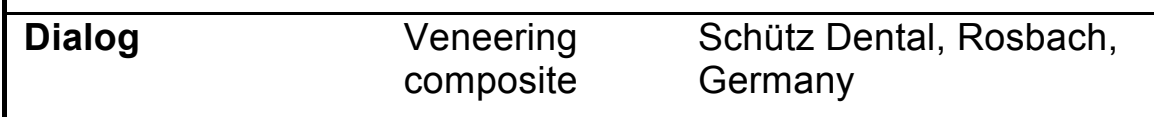

Inorganic fillers (74 w\% 66 Vol.\%)

Glass fillers (particle size: $0.7 \mu \mathrm{m}$ )

Pyrogene orthosilisic acid (average particle size: $0.04 \mu \mathrm{m})$

Monomer $(25 \mathrm{w} \%)$

Urethandimethacrylate

Butandioldimethacrylate

Bis-GMA

Initiators, stabilizers, pigments

Table 1. The brand, type, manufacturer, and chemical composition of the main materials used in this study. 


\begin{tabular}{|c|c|c|c|c|c|c|c|c|c|}
\hline & \multicolumn{2}{|c|}{ RelyX ARC } & \multicolumn{2}{|c|}{ Bifix DC } & \multicolumn{2}{|c|}{ Variolink II } & \multicolumn{2}{|c|}{ Multilink } & Total \\
\hline & \multicolumn{9}{|c|}{ Pontic Type } \\
\hline & Centra & ateral & Centra & Lateral & Central & Lateral & Centra & Lateral & \\
\hline Mandible & 19 & 21 & 11 & 13 & 7 & 11 & 13 & 17 & 112 \\
\hline Maxilla & 9 & 12 & 10 & 11 & 6 & 8 & 3 & 4 & 63 \\
\hline Total & 28 & 33 & 21 & 24 & 13 & 19 & 16 & 21 & 175 \\
\hline
\end{tabular}

Table. 2. Distribution and location of the FRC FDPs cemented using 4 types of resin cement. 


\section{Legends:}
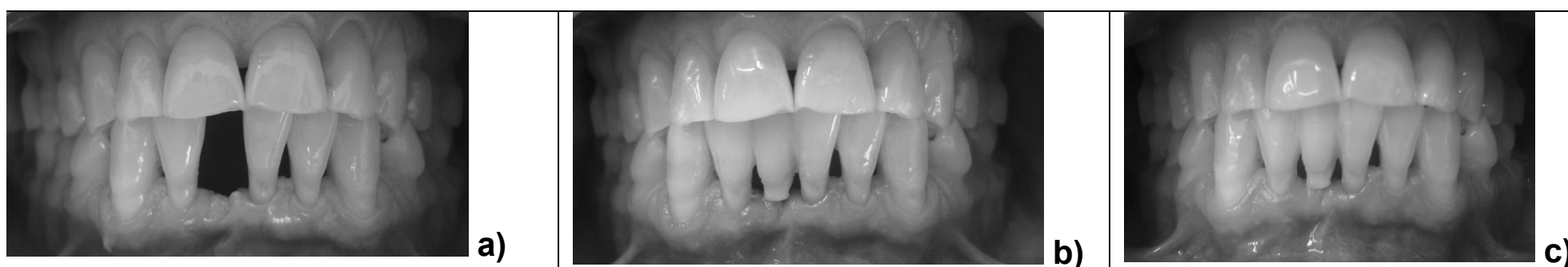

c)

Figs. 1a-c Representative photos of a) Missing 41, b) indirect FRC FDP replacing the missing tooth at baseline, c) FRC FDP at 3 years follow up. 


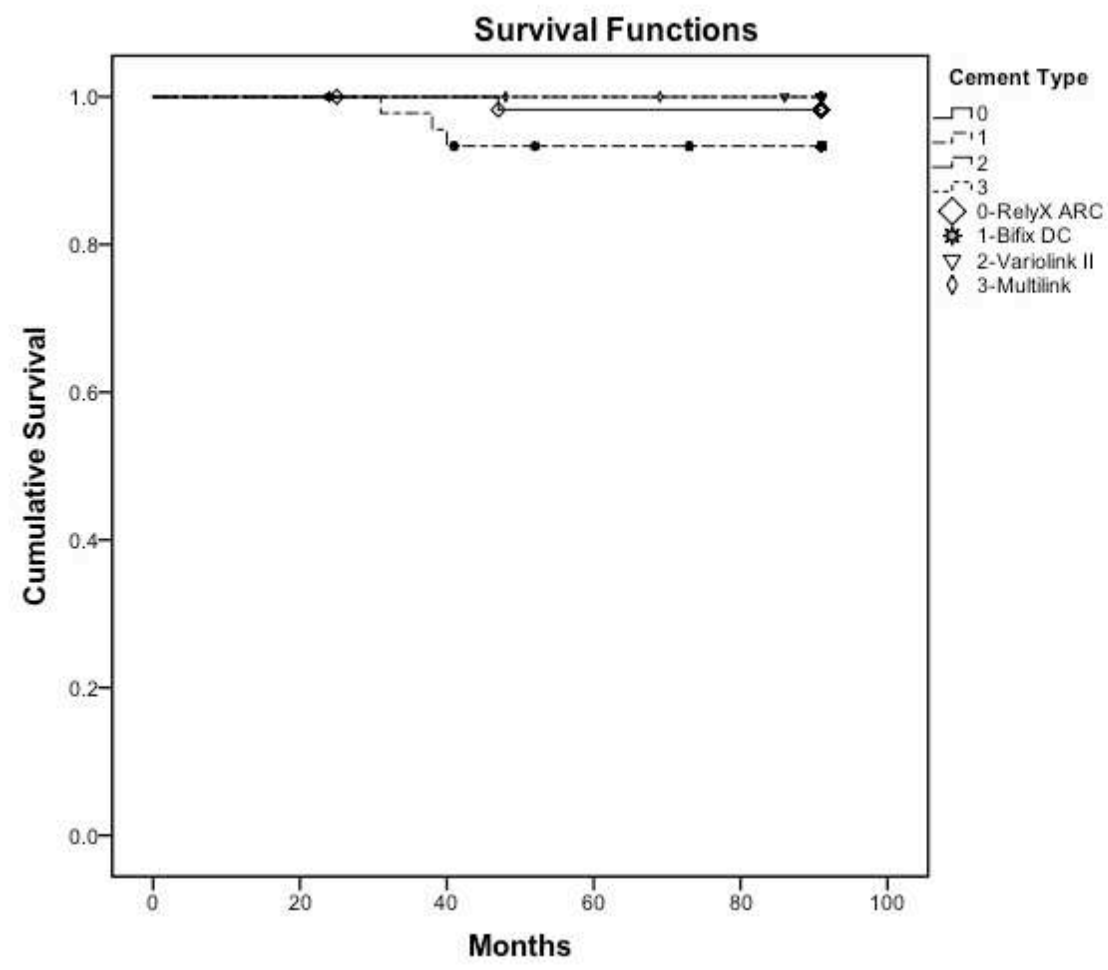

Fig. 2 Event-free survival rates of surface-retained, anterior FRC FDPs based on the resin cement type. (RelyX ARC, $n=61$, Bifix DC, $n=45$, Variolink II, $n=32$, Multilink, $n=37$ ). 